\title{
On Janowski Analytic $(p, q)$-Starlike Functions in Symmetric Circular Domain
}

\author{
Muhammad Ghaffar Khan, ${ }^{1}$ Bakhtiar Ahmad, ${ }^{1}$ Basem Aref Frasin, ${ }^{2}$ \\ and Thabet Abdeljawad ${ }^{3,4,5}$ \\ ${ }^{1}$ Department of Mathematics, Abdul Wali Khan University Mardan, Pakistan \\ ${ }^{2}$ Faculty of Science, Department of Mathematics, Al al-Bayt University, Mafraq, Jordan \\ ${ }^{3}$ Department of Mathematics and General Sciences, Prince Sultan University, P.O. Box 66833, Riyadh 11586, Saudi Arabia \\ ${ }^{4}$ Department of Medical Research, China Medical University, Taichung 40402, Taiwan \\ ${ }^{5}$ Department of Computer Science and Information Engineering, Asia University, Taichung, Taiwan
}

Correspondence should be addressed to Thabet Abdeljawad; tabdeljawad@psu.edu.sa

Received 30 June 2020; Revised 19 September 2020; Accepted 23 September 2020; Published 10 October 2020

Academic Editor: Serkan Araci

Copyright (c) 2020 Muhammad Ghaffar Khan et al. This is an open access article distributed under the Creative Commons Attribution License, which permits unrestricted use, distribution, and reproduction in any medium, provided the original work is properly cited.

The main object of the present paper is to apply the concepts of $(p, q)$-derivative by establishing a new subclass of analytic functions connected with symmetric circular domain. Further, we investigate necessary and sufficient conditions for functions belonging to this class. Convex combination, weighted mean, arithmetic mean, growth theorem, and convolution property are also determined.

\section{Introduction and Definitions}

Quantum calculus or $q$-calculus is a generalization of classical calculus without the notation of limits. The theory of $q$ -calculus is established by Jackson, for details see $[1,2]$. Due to its numerous applications in various branches of applied sciences and mathematics, for example, physics, operator theory, numerical analysis, and differential equations, attracted researchers to this field. A detailed study on applications of $q$-calculus in operator theory may be found in [3]. The geometric interpretation of $q$-calculus has been recognized through studies on quantum groups. Starlikeness and convexity are two major properties of analytic functions. Ismail et al. [4] investigated the generalized starlike function $\mathcal{S}^{*}$, and certain subclasses close-to-convex functions of $q$-Mittag-Leffler functions were studied by Srivastava and Bansal [5], also the reader is referred to [6-12] for more details.

The foundation of quantum calculus is on one parameter, while the postquantum calculus or simply $(p, q)$-calculus is the generalization of $q$-calculus based on two parameters. By setting $p=1$ in $(p, q)$-calculus, the $q$-calculus is obtained.
The $(p, q)$-integer was considered by Chakrabarti and Jagannathan [13], also see the work [14-18]. The idea of $q$-starlike is extended to $(p, q)$-stalikeness by Raza et al. [19]. Before we define our new class in this field, we give some basics for a better understanding of the work to follow.

Let $\mathscr{A}$ represent the family of function $f$ that are analytic in the open unit disc $\mathfrak{D}=\{z \in \mathbb{C}:|z|<1\}$ having the series expansion

$$
f(z)=z+\sum_{n=2}^{\infty} a_{n} z^{n},(z \in \mathfrak{D})
$$

A function $f(z)$ of the form (1) is subordinate to function $g(z)=z+\sum_{n=2}^{\infty} b_{n} z^{n}$, symbolically represented $f(z)<g(z)$, if there occur a Schwarz function $w(z)$ with limitation that $w$ $(0)=0$, and $|w(z)| \leq 1$, then $f(z)=g(w(z))$. While the convolution of these functions can be defined by

$$
f(z) * g(z)=z+\sum_{n=2}^{\infty} a_{n} b_{n} z^{n},(z \in \mathfrak{D})
$$


For $0<q<1$, the $q$-derivative of a function $f$ is defined by

$$
\partial_{q} f(z)=\frac{f(z)-f(q z)}{z(1-q)},(z \neq 0, q \neq 1)
$$

where

$$
[n]_{q}=\frac{1-q^{n}}{1-q}=1+\sum_{l=1}^{n-1} q^{l}, \quad[0, q]=0
$$

see [13] for details.

Also for $0<p<q<1$, the $(p, q)$-derivative of a function $f$ is defined in [2] as

$$
\partial_{p, q} f(z)=\frac{f(p z)-f(q z)}{z(p-q)},(z \neq 0, p \neq q) .
$$

It can easily be seen that for $n \in \mathbb{N}:=\{1,2,3, \cdots\}$ and $z$ $\in \mathfrak{D}, \partial_{p, q}\left(\sum_{n=1}^{\infty} a_{n} z^{n}\right)=\sum_{n=1}^{\infty}[n]_{p, q} a_{n} z^{n-1}$,

where

$$
[n]_{p, q}=\frac{p^{n}-q^{n}}{p-q}
$$

We note that $\partial_{1, q} f(z)=\partial_{q} f(z)$ (for more on this topic one should read [20-22]).

Sakaguchi [23], in year 1956, established the class of starlike functions with respect to symmetrical points denoted by $\mathcal{S}_{s}^{*}$ of holomorphic univalent functions in $\mathfrak{D}$ if the below condition is satisfies

$$
\operatorname{Re} \frac{2 z f^{\prime}(z)}{f(z)-f(-z)}>0,(z \in \mathfrak{A})
$$

Motivated by the work of $[19,23,24]$, we now define $\mathcal{S}_{p, q}^{*}(l, m, \mathscr{C}, \mathscr{D})$ given below.

Definition 1. Let $-\mathscr{D} \leq \mathscr{C}<\mathscr{D} \leq 1,0<p<q \leq 1$ and $-1 \leq m$ $<l \leq 1$, then the function $f \in \mathscr{A}$ is in the class $\mathcal{S}_{p, q}^{*}(l, m, \mathscr{C}$, D) if it satisfies

$$
\frac{(l-m) z \partial_{p, q} f(z)}{f(l z)-f(m z)} \prec \frac{1+\mathscr{C} z}{1+\mathscr{D} z},(z \in \mathfrak{D}),
$$

where the symbol “ $\prec$ indicates the well-known subordination.

We note that $\mathcal{S}_{1, q}^{*}(l, m, \mathscr{C}, \mathscr{D})=\mathcal{S}_{q}^{*}(l, m, \mathscr{C}, \mathscr{D})$, where $\mathcal{S}_{q}^{*}(l, m, \mathscr{C}, \mathscr{D})=\left\{f \in \mathscr{A}: \frac{(l-m) z \partial_{q} f(z)}{f(l z)-f(m z)} \prec \frac{1+\mathscr{C} z}{1+\mathscr{D} z},(z \in \mathfrak{D})\right\}$, and

$$
\begin{aligned}
\lim _{q \rightarrow 1^{-}} \mathcal{S}_{q}^{*}(1,-1, \mathscr{C}, \mathscr{D})=\mathcal{S}^{*}(\mathscr{C}, \mathscr{D}) \\
\quad=\left\{f \in \mathscr{A}: \frac{2 z f^{\prime}(z)}{f(z)-f(-z)} \prec \frac{1+\mathscr{C} z}{1+\mathscr{D} z},(z \in \mathfrak{D})\right\} .
\end{aligned}
$$

Equivalently, a function $f \in \mathscr{A}$ is in the $\mathcal{S}_{p, q}^{*}(l, m, \mathscr{C}, \mathscr{D})$ if and only if

$$
\left|\frac{(l-m) z \partial_{p, q} f(z) / f(l z)-f(m z)-1}{\mathscr{D}\left((l-m) z \partial_{p, q} f(z) / f(l z)-f(m z)\right)-\mathscr{C}}\right|<1,(z \in \mathfrak{D}) .
$$

In our main results, in the next section, we evaluate the criteria for functions belonging to this newly defined class. After that, the convex combination property for this class will be discussed. Then utilizing these results, the weighted mean and arithmetic mean properties will be investigated. Further, convolution type results will be discussed in the form of two theorems. At the end of this article, a conclusion and future work will be presented.

\section{Main Results}

Theorem 2. Let $f \in \mathscr{A}$ be of the form (1). Then the function $f \in \mathcal{S}_{p, q}^{*}(l, m, \mathscr{C}, \mathscr{D})$, if and only if the following inequality holds

$$
\sum_{n=2}^{\infty}\left\{[n]_{p, q}(1+\mathscr{D})-(\mathscr{C}+1) \frac{l^{n}-m^{n}}{l-m}\right\}\left|a_{n}\right|<(\mathscr{D}-\mathscr{C}) .
$$

Proof. Let us suppose that the first inequality (12) holds. Then to show that $f \in \mathcal{S}_{p, q}^{*}(l, m, \mathscr{C}, \mathscr{D})$, we only need to prove the inequality (11). For this consider

$$
\begin{aligned}
& \left|\frac{(l-m) z \partial_{p, q} f(z) / f(l z)-f(m z)-1}{D\left((l-m) z \partial_{p, q} f(z) / f(l z)-f(m z)\right)-C}\right| \\
& \quad=\left|\frac{\sum_{n=2}^{\infty}\left[[n]_{p, q}-l^{n}-m^{n} / l-m\right] \alpha_{n} z^{n}}{(D-C) z-\sum_{n=2}^{\infty}\left[D[n]_{p, q}-C\left(l^{n}-m^{n} / l-m\right) \alpha_{n} z^{n}\right]}\right| \\
& \quad \leq \frac{\sum_{n=2}^{\infty}\left[[n]_{p, q}-l^{n}-m^{n} / l-m\right]\left|\alpha_{n}\right|}{(D-C)-\sum_{n=2}^{\infty}\left[D[n]_{p, q}-C\left(l^{n}-m^{n} / l-m\right)\right]\left|\alpha_{n}\right|}<1,
\end{aligned}
$$

where we used and this completes the direct part. Conversely, let $f \in \mathcal{S}_{p, q}^{*}(l, m, \mathscr{C}, \mathscr{D})$ be of from (1). Then from (11), we have for $z \in \mathfrak{D}$, 


$$
\begin{aligned}
& \left|\frac{(l-m) z \partial_{p, q} f(z) / f(l z)-f(m z)-1}{D\left((l-m) z \partial_{p, q} f(z) / f(l z)-f(m z)\right)-C}\right| \\
& \quad=\left|\frac{\sum_{n=2}^{\infty}\left[[n]_{p, q}-l^{n}-m^{n} / l-m\right]\left|\alpha_{n} z^{n}\right|}{(D-C) z-\sum_{n=2}^{\infty}\left[D[n]_{p, q}-C\left(l^{n}-m^{n} / l-m\right)\right]\left|\alpha_{n} z^{n}\right|}\right|<1
\end{aligned}
$$

Since $|\operatorname{Rez}|<|z|<1$, we have

$\operatorname{Re}\left\{\frac{\sum_{n=2}^{\infty}\left[[n]_{p, q}-l^{n}-m^{n} / l-m\right]\left|\alpha_{n} z^{n}\right|}{(D-C) z-\sum_{n=2}^{\infty}\left[D[n]_{p, q}-C\left(l^{n}-m^{n} / l-m\right)\right]\left|\alpha_{n} z^{n}\right|}\right\}<1$

Now we choose values of $z$ on the real axis such that $(l-m) z \partial_{p, q} f(z) / f(l z)-f(m z)$ is real. Upon clearing the denominator in (15) and letting $z \longrightarrow 1^{-}$through real values, we obtain the required inequality (12).

Theorem 3. Let $f_{i} \in \mathcal{S}_{p, q}^{*}(l, m, \mathscr{C}, \mathscr{D})$ and having power series representations

$$
f_{i}(z)=z+\sum_{k=1}^{\infty} a_{k, i} z^{k}, \text { for } i=1,2,3, \cdots, t
$$

Then $\Phi \in \mathcal{S}_{p, q}^{*}(l, m, \mathscr{C}, \mathscr{D})$, where

$$
\Phi(z)=\sum_{i=1}^{t} \omega_{i} f_{i}(z) \text { with } \sum_{i=1}^{t} \omega_{i}=1
$$

Proof. By Theorem 2, one can write

$$
\sum_{n=2}^{\infty}\left\{\frac{\left.[n]_{p, q}(1+\mathscr{D})-(\mathscr{C}+1)\left(l^{n}-m^{n} / l-m\right)\right]}{(\mathscr{D}-\mathscr{C})}\right\}\left|a_{n, i}\right|<1 .
$$

Therefore

$$
\begin{aligned}
\Phi(z) & =\sum_{i=1}^{t} \omega_{i}\left(z+\sum_{n=2}^{\infty} a_{n, i} z^{n}\right) \\
& =z+\sum_{i=1}^{t} \sum_{n=2}^{\infty} \omega_{i} a_{n, i} z^{n} \\
& =z+\sum_{n=2}^{\infty}\left(\sum_{i=1}^{t} \omega_{i} a_{n, i}\right) z^{n}
\end{aligned}
$$

however,

$$
\begin{aligned}
\sum_{n=2}^{\infty} & \frac{\left[[n]_{p, q}(1+\mathscr{D})-(\mathscr{C}+1)\left(l^{n}-m^{n} / l-m\right)\right]}{(\mathscr{D}-\mathscr{C})}\left(\left|\sum_{i=1}^{t} \omega_{i} a_{n, i}\right|\right) \\
& =\sum_{i=1}^{t} \omega_{i}\left[\sum_{n=2}^{\infty}, \frac{\left[[n]_{p, q}(1+\mathscr{D})-(\mathscr{C}+1)\left(l^{n}-m^{n} / l-m\right)\right]}{(\mathscr{D}-\mathscr{C})},\left|a_{n, i}\right|\right] \leq 1,
\end{aligned}
$$

then $\Phi \in \mathcal{S}_{p, q}^{*}(l, m, \mathscr{C}, \mathscr{D})$. Hence, the proof is completed.

Theorem 4. If $f_{1}, f_{2} \in \mathcal{S}_{p, q}^{*}(l, m, \mathscr{C}, \mathscr{D})$, then their weighted mean $\psi_{k}$ is also in $\mathcal{S}_{p, q}^{*}(l, m, \mathscr{C}, \mathscr{D})$, where $\psi_{k}$ is defined by

$$
\psi_{k}(z)=\left\{\frac{(1-k) f_{1}(z)+(1+k) f_{2}(z)}{2}\right\} .
$$

Proof. From (21), one can easily write

$$
\psi_{k}(z)=z+\sum_{n=2}^{\infty}\left\{\frac{(1-k) a_{n}+(1+k) b_{n}}{2}\right\} z^{n}
$$

To prove that $\psi_{k} \in \mathcal{S}_{p, q}^{*}(l, m, \mathscr{C}, \mathscr{D})$, it is enough to show that

$$
\begin{gathered}
\sum_{n=2}^{\infty}\left\{\frac{\left[[n]_{p, q}(1+\mathscr{D})-(\mathscr{C}+1)\left(l^{n}-m^{n} / l-m\right)\right]}{(\mathscr{D}-\mathscr{C})}\right\} \\
\quad\left\{\frac{(1-k) a_{n}+(1+k) b_{n}}{2}\right\}<1 .
\end{gathered}
$$

For this, consider

$$
\begin{aligned}
\sum_{n=2}^{\infty}\left\{\frac{\left[[n]_{p, q}(1+\mathscr{D})-(\mathscr{C}+1)\left(l^{n}-m^{n} / l-m\right)\right]}{(\mathscr{D}-\mathscr{C})}\right\} \\
\quad\left\{\frac{(1-k) a_{n}+(1+k) b_{n}}{2}\right\} \\
=\frac{(1-j)}{2} \cdot \sum_{n=2}^{\infty}\left\{\frac{\left[[n]_{p, q}(1+\mathscr{D})-(\mathscr{C}+1)\left(l^{n}-m^{n} / l-m\right)\right]}{(\mathscr{D}-\mathscr{C})}\right\}\left|a_{n}\right| \\
\quad+\frac{(1+j)}{2} \cdot \sum_{n=2}^{\infty}\left\{\frac{\left[[n]_{p, q}(1+\mathscr{D})-(\mathscr{C}+1)\left(l^{n}-m^{n} / l-m\right)\right]}{(\mathscr{D}-\mathscr{C})}\right\}\left|b_{n}\right| \\
<\frac{(1-k)}{2}+\frac{(1+k)}{2}=1,
\end{aligned}
$$

where we have used inequality (12). Which completes the proof. 
Theorem 5. Let $f_{i} \in \mathcal{S}_{p, q}^{*}(l, m, \mathscr{C}, \mathscr{D})$, with $i=1,2, \cdots, j$. Then, their arithmetic mean $\varphi$ of $f_{i}$

$$
\varphi(z)=\frac{1}{j} \sum_{i=1}^{j} f_{i}(z)
$$

is also in the class $\mathcal{S}_{p, q}^{*}(l, m, \mathscr{C}, \mathscr{D})$.

Proof. From (25), we can write

$$
\varphi(z)=\frac{1}{j} \sum_{i=1}^{j}\left(z+\sum_{n=2}^{\infty} a_{n, i} z^{n}\right)=z+\sum_{n=2}^{\infty}\left(\frac{1}{j} \sum_{i=1}^{j} a_{n, i}\right) z^{n} .
$$

Since $f_{i} \in \mathcal{S}_{p, q}^{*}(l, m, \mathscr{C}, \mathscr{D})$ for every $i=1,2, \cdots, j$, using (12), we have

$$
\begin{aligned}
& \sum_{n=2}^{\infty}\left\{\frac{\left.[n]_{p, q}(1+\mathscr{D})-(\mathscr{C}+1)\left(l^{n}-m^{n} / l-m\right)\right]}{(\mathscr{D}-\mathscr{C})}\right\} \cdot\left|\frac{1}{j} \sum_{i=1}^{j} a_{n, i}\right| \\
& \quad=\frac{1}{j} \sum_{i=1}^{j}\left(\sum_{n=2}^{\infty}\left\{\frac{\left[[n]_{p, q}(1+\mathscr{D})-(\mathscr{C}+1)\left(l^{n}-m^{n} / l-m\right)\right]}{(\mathscr{D}-\mathscr{C})}\right\} \cdot\left|a_{n, i}\right|\right) \\
& \quad \leq \frac{1}{j} \sum_{i=1}^{j}(1)=1,
\end{aligned}
$$

which complete the proof.

Theorem 6. Let $f \in \mathcal{S}_{p, q}^{*}(l, m, \mathscr{C}, \mathscr{D})$. Then for $|z|=r, 0<r<1$,

$$
r-\delta_{p, q}(l, m, \mathscr{C}, \mathscr{D}) r^{2}<|f(z)|<r+\delta_{p, q}(l, m, \mathscr{C}, \mathscr{D}) r^{2}
$$

where

$$
\begin{gathered}
\delta_{p, q}(l, m, \mathscr{C}, \mathscr{D})=\frac{(\mathscr{C}-\mathscr{D})}{[2]_{p, q}(1-\mathscr{D})+(\mathscr{C}+1)(l+m)} \\
r-\gamma_{p, q}(l, m, \mathscr{C}, \mathscr{D}) r^{2}<\left|\partial_{p, q} f(z)\right|<r+\gamma_{p, q}(l, m, \mathscr{C}, \mathscr{D}) r^{2},
\end{gathered}
$$

where

$$
\gamma_{p, q}(l, m, \mathscr{C}, \mathscr{D})=\frac{(\mathscr{C}-\mathscr{D})}{(1-\mathscr{D})+(\mathscr{C}+1)(l+m)} .
$$

Proof. To prove (28), consider

$$
|f(z)| \leq r+\sum_{n=2}^{\infty}\left|a_{n}\right||r|^{n}
$$

as $0<r<1$ so $r^{n}<r^{2}$ hence

$$
|f(z)|<r+r^{2} \sum_{n=2}^{\infty}\left|a_{n}\right| \leq r+\frac{(\mathscr{C}-\mathscr{D})}{[2]_{p, q}(1-\mathscr{D})+(\mathscr{C}+1)(l+m)} r^{2} .
$$

Similarly,

$$
\begin{aligned}
|f(z)| & \geq r-\sum_{n=2}^{\infty}\left|a_{n}\right||r|^{n}>r-r^{2} \sum_{n=2}^{\infty}\left|a_{n}\right| \\
& \geq r-\frac{(\mathscr{C}-\mathscr{D})}{[2]_{p, q}(1-\mathscr{D})+(\mathscr{C}+1)(l+m)} r^{2} .
\end{aligned}
$$

Hence complete the proof of (28). Similarly, we can prove (30).

Theorem 7. Let $f_{i} \in \mathcal{S}_{p, q}^{*}(l, m, \mathscr{C}, \mathscr{D})$, such that

$$
f_{i}(z)=z+\sum_{n=2}^{\infty} a_{n, i} z^{n}, i=1,2
$$

with condition $\left|a_{n, 2}\right| \leq 1$, then $f_{1} * f_{2} \in \mathcal{S}_{p, q}^{*}(l, m, \mathscr{C}, \mathscr{D})$.

Proof. Since form (35), we have

$$
f_{i}(z)=z+\sum_{n=2}^{\infty} a_{n, i} z^{n}, i=1,2
$$

Then convolution is defined as

$$
\left(f_{1} * f_{2}\right)(z)=z+\sum_{n=2}^{\infty} a_{n, 1} a_{n, 2} z^{n}
$$

Since $f_{2} \in \mathcal{S}_{p, q}^{*}(l, m, \mathscr{C}, \mathscr{D})$, with limitation that $\left|a_{n, 2}\right| \leq 1$. Therefore

$$
\begin{aligned}
& \sum_{n=2}^{\infty}\left\{\frac{\left.[n]_{p, q}(1+\mathscr{D})-(\mathscr{C}+1)\left(l^{n}-m^{n} / l-m\right)\right]}{(\mathscr{D}-\mathscr{C})}\right\}\left|a_{n, 1}\right|\left|a_{n, 2}\right| \\
& \quad \leq \sum_{n=2}^{\infty}\left\{\frac{\left[[n]_{p, q}(1+\mathscr{D})-(\mathscr{C}+1)\left(l^{n}-m^{n} / l-m\right)\right]}{(\mathscr{D}-\mathscr{C})}\right\}\left|a_{n, 1}\right|<1 .
\end{aligned}
$$

Hence $f_{1} * f_{2} \in \mathcal{S}_{p, q}^{*}(l, m, \mathscr{C}, \mathscr{D})$.

Theorem 8. Let $f(z) \in \mathcal{S}_{p, q}^{*}(l, m, \mathscr{C}, \mathscr{D})$. Then

$$
\frac{1}{z}\left[f(z) *\left(\frac{\left(1+\mathscr{D} e^{i \theta}\right) z}{(1-p z)(1-q z)}-\frac{\left(1+\mathscr{C} e^{i \theta}\right) z}{(1-l z)(1-m z)}\right)\right] \neq 0 .
$$


Proof. Let $f(z) \in \mathcal{S}_{p, q}^{*}(l, m, \mathscr{C}, \mathscr{D})$. Then by definition of subordination, there exists a Schwarz function $w(z)$, such that $w(0)=0$ and $|w(z)|<1$,

$$
\frac{(l-m) z \partial_{p, q} f(z)}{f(l z)-f(m z)}=\frac{1+\mathscr{C} w(z)}{1+\mathscr{D} w(z)},
$$

equivalently,

$$
\begin{aligned}
& \frac{(l-m) z \partial_{p, q} f(z)}{f(l z)-f(m z)} \neq \frac{1+\mathscr{C} e^{i \theta}}{1+\mathscr{D} e^{i \theta}}, \\
& z \partial_{p, q} f(z)\left(1+\mathscr{D} e^{i \theta}\right)-\frac{f(l z)-f(m z)}{l-m}\left(1+\mathscr{C} e^{i \theta}\right) \neq 0,
\end{aligned}
$$

using the relations

$$
\begin{gathered}
z \partial_{p, q} f(z)=f(z) * \frac{z}{(1-p z)(1-q z)}, \\
\frac{f(l z)-f(m z)}{l-m}=f(z) *\left[\frac{z}{(1-l z)(1-m z)}\right],
\end{gathered}
$$

now (42), becomes

$$
\frac{1}{z}\left[f(z) *\left(\frac{\left(1+\mathscr{D} e^{i \theta}\right) z}{(1-p z)(1-q z)}-\frac{\left(1+\mathscr{C} e^{i \theta}\right) z}{(1-l z)(1-m z)}\right)\right] \neq 0,
$$

which completes the proof.

\section{Conclusions}

Utilizing the concepts of postquantum calculus, we defined a new subclass of analytic functions associated with symmetric circular domain. For this class, we investigated some useful results such as necessary and sufficient problem, convex combination, weight mean, arithmetic mean, distortion bounds, and convolution property. There are some problems open for researchers such as radii problems, extreme point theorem, analytic criteria, and integral mean of inequality. Moreover, this concept is new and can be extended to meromorphic functions and harmonic functions.

\section{Data Availability}

Data used to support the findings of this study are included within the article.

\section{Conflicts of Interest}

The authors declare that they have no competing interests.

\section{Authors' Contributions}

All authors jointly worked on the results and they read and approved the final manuscript.

\section{Acknowledgments}

The authors would like to express their sincere thanks to the reviewers for their helpful comments and suggestions. This work is supported by the Department of Mathematics and General Sciences, Prince Sultan University, P.O. Box 66833, Riyadh 11586, Saudi Arabia.

\section{References}

[1] D. O. Jackson, T. Fukuda, O. Dunn, and E. Majors, "On q -definite integrals," Quarterly Journal of Pure and Applied Mathematics, vol. 41, pp. 193-203, 1910.

[2] F. H. Jackson, "XI.-On $q$-Functions and a certain difference operator," Transactions of The Royal Society of Edinburgh, vol. 46, no. 2, pp. 253-281, 1909.

[3] A. Aral, V. Gupta, and R. P. Agarwal, Applications of q-calculus in operator theory, Springer, New York, 2013.

[4] M. E. H. Ismail, E. Merkes, and D. Styer, "A generalization of starlike functions," Complex Variables, Theory and Application: An International Journal, vol. 14, no. 1-4, pp. 77-84, 1990.

[5] H. M. Srivastava and D. Bansal, "Close-to-convexity of a certain family of $q$-Mittag-Leffler functions," Journal of Nonlinear and Variational Analysis, vol. 1, pp. 61-69, 2017.

[6] G. Yasmin, A. Muhyi, and S. Araci, "Certain results of q -Sheffer-Appell Polynomials," Symmetry, vol. 11, no. 2, p. 159, 2019.

[7] H. M. Srivastava, G. Yasmin, A. Muhyi, and S. Araci, "Certain results for the Twice-Iterated 2D q-Appell Polynomials," Symmetry, vol. 11, no. 10, p. 1307, 2019.

[8] S. D. Purohit and R. K. Raina, "Certain subclasses of analytic functions associated with fractional q-calculus operators," Mathematica Scandinavica, vol. 109, no. 1, pp. 55-70, 2011.

[9] L. Shi, M. Ghaffar Khan, and B. Ahmad, "Some geometric properties of a family of analytic functions involving a generalized q-operator," Symmetry, vol. 12, no. 2, p. 291, 2020.

[10] S. D. Purohit, "A new class of multivalently analytic functions associated with fractional q-calculus operators," Fractional Differential Calculus, vol. 2, no. 2, pp. 129-138, 2012.

[11] S. D. Purohit and R. K. Raina, "Some classes of analytic and multivalent functions associated with q-derivative operators," Acta Universitatis Sapientiae, Mathematica, vol. 6, no. 1, pp. 5-23, 2014

[12] S. D. Purohit and F. Ucar, "An application of q-Sumudu transform for fractional $q$-kinetic equation," Turkish Journal of Mathematics, vol. 42, no. 2, pp. 726-734, 2018.

[13] R. Chakrabarti and R. Jagannathan, "A (p, q)-oscillator realization of two-parameter quantum algebras," Journal of Physics A: Mathematical and General, vol. 24, no. 13, pp. L711-L718, 1991.

[14] J. D. Bukweli-Kyemba and M. N. Hounkonnou, "Quantum deformed algebra: coherent states and special functions," 2013, https://arxiv.org/abs/1301.0116.

[15] B. A. Frasin and G. Murugusundaramoorthy, "A subordination results for a class of analytic functions defined by q-differential operator," Annales Universitatis Paedagogicae Cracoviensis. Studia Mathematica, vol. 19, no. 1, pp. 53-64, 2020.

[16] T. Al-Hawary, F. Yousef, and B. A. Frasin, "Subclasses of analytic functions of complex order involving Jackson's (p, q) 
-derivative," Proceedings of International Conference on Fractional Differentiation and its Applications (ICFDA), 2018.

[17] C. Ramachandran, T. Soupramanien, and B. A. Frasin, "New subclasses of analytic function associated with q-difference operator," European Journal of Pure and Applied Mathematics, vol. 10, no. 2, pp. 348-362, 2017.

[18] P. N. Sadjang, "On the foundamental theorem of $(p, q)$-calculus and some $(p, q)$-Taylor formulas," 2013, https://arxiv .org/abs/1309.3934.

[19] N. Raza, E. S. A. AbuJarad, G. Srivastava, H. M. Srivastava, and M. H. AbuJarad, "Fekete-Szego inequality for classes of (p, q) -starlike and (p, q)-convex functions," 2019, https://arxiv .org/abs/1912.05298.

[20] M. K. Aouf and T. M. Seoudy, "Convolution properties for classes of bounded analytic functions with complex order defined by $\backslash(\mathrm{q}-\backslash)$ derivative operator," Revista de la Real Academia de Ciencias Exactas, Físicas y Naturales. Serie A. Matemáticas, vol. 113, no. 2, pp. 1279-1288, 2019.

[21] T. M. Seoudy and M. K. Aouf, "Convolution properties for certain classes of analytic functions defined by $q$-Derivative operator," Abstract and Applied Analysis, vol. 2014, Article ID 846719, 7 pages, 2014.

[22] T. M. Seoudy and M. K. Aouf, "Coefficient estimates of new classes of $q$-starlike and $q$-convex functions of complex order," Journal of Mathematical Inequalities, vol. 10, no. 1, pp. 135145, 2007.

[23] K. Sakaguchi, "On a certain univalent mapping," Journal of the Mathematical Society of Japan, vol. 2, pp. 72-75, 1959.

[24] S. Kavitha, N. E. Cho, and G. Murugusundaramoorthy, "On (p, q)-quantum calculus involving quasi-subordination," in Advances in Algebra and Analysis, Trends in Mathematics, V. Madhu, A. Manimaran, D. Easwaramoorthy, D. Kalpanapriya, and M. Mubashir Unnissa, Eds., pp. 215-223, Birkhäuser, Cham, 2018. 